\title{
Leptospirosis: challenges in diagnosis, and predictors of severity
}

\author{
Orator: Rajapakse $\mathbf{S}^{1}$
}

Co-authors: Fernando $\mathrm{N}^{2}$, Niloofa MJR${ }^{2}$, de Silva $\mathrm{HJ}^{3}$, Karunanayake $\mathrm{L}^{4}$, Premawansa $\mathrm{S}^{4}$, Handunnetti SM${ }^{2}$

Journal of the Ceylon College of Physicians, 2016, 47, 60-69

DOI: http://doi.org/10.4038/jccp.v47i2.7785

\section{Introduction}

Leptospirosis is a ubiquitous zoonosis which results in high morbidity and considerable mortality in humans. ${ }^{1}$ On a global scale it is estimated that nearly 10,000 cases of severe leptospirosis are hospitalized annually worldwide. ${ }^{2}$ Rodents, marsupials, or any mammal has the potential to be the animal reservoir. The organisms are excreted to the environment via the urine of the animal host. Transmission to humans can be direct (inoculation via tissues, body fluids and urine of infected animals) or indirect (entry via mucosal surfaces or breached skin from sources contaminated with the causative agent). ${ }^{2}$

This oration presented the findings of a series of on-going prospective studies focusing on the evaluation of rapid diagnostic tests for leptospirosis, the clinical and laboratory correlates of leptospirosis and their relationship to outcome in leptospirosis, and the possible place of biomarkers in predicting the development of severe disease. In particular, the role of oxidative stress in the pathogenesis of the disease was examined, with the ultimate aim of identifying potential points for interventions which could modify the course of the disease. The work presented here is the result of the efforts of a large team of researchers from the Universities of Colombo and Kelaniya, the Medical Research Institute, and the collaborating hospitals.

\section{Epidemiology of leptospirosis}

An accurate assessment of the disease burden of leptospirosis is not easy due to diagnostic difficulties and

\footnotetext{
${ }^{1}$ Department of Clinical Medicine, Faculty of Medicine, University of Colombo, Sri Lanka.

${ }^{2}$ Institute of Biochemistry, Molecular Biology and Biotechnology, University of Colombo, Sri Lanka

${ }^{3}$ Department of Medicine, Faculty of Medicine, University of Kelaniya, Ragama, Sri Lanka.

${ }^{4}$ Medical Research Institute, Colombo, Sri Lanka

${ }^{5}$ Department of Zoology, Faculty of Science, University of Colombo, Sri Lanka.
}

Corresponding author: SR

E-mail: senaka@med.cmb.ac.lk under-reporting. Leptospirosis is often a clinical diagnosis, and the standard diagnostic test is the Microscopic Agglutination Test (MAT). In many clinical settings, leptospirosis is only a presumed diagnosis, made based on clinical features, and there can be a considerable overlap with other mimics such as dengue, hanta virus infections and bacterial sepsis.

In Sri Lanka, data from the Epidemiology Unit of the Ministry of Health shows that leptospirosis has emerged as a key infection over the last 20 years. From 167 cases in 1991, the numbers have swelled to 4545 cases in $2010 .^{3}$ The highest number of cases (7099) was reported in 2008 when there was an epidemic of leptospirosis (Figure 1).

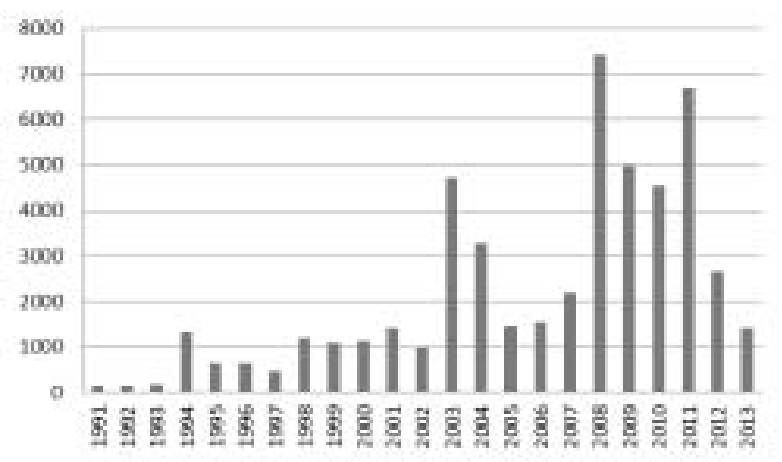

Figure 1. Suspected cases of leptospirosis in Sri Lanka: 1991- 2013 (Epidemiology Unit, 2013).

\section{Clinical manifestations of leptospirosis in humans}

The clinical presentation of leptospirosis shows wide variation. Most patients develop a simple uncomplicated febrile illness, while some go on to develop a more severe form. Severe disease can affect many organ systems resulting in acute kidney injury, hepatitis, pulmonary haemorrhage, disseminated intravascular coagulation, meningitis, and multi-organ dysfunction syndrome. Many atypical manifestations are known to occur. ${ }^{4}$ The mortality in severe disease varies in different case series, but can be as high as $40 \% .^{5}$ The most difficult challenge faced by the treating clinician is to predict, at the point of admission, which patients will have a complicated 
course. Despite attempts to retrospectively correlate mortality and severity with clinical variables and investigation findings, no scoring systems or criteria exist which can predict outcome in leptospirosis. ${ }^{6}$

\section{Management of leptospirosis}

The management of leptospirosis largely comprises supportive care. Antibiotics are administered; penicillin, doxycycline and cephalosporins are generally effective, although a recent Cochrane Review raises doubts as to the effectiveness of antibiotics. ${ }^{7}$ Renal support in the form of dialysis is often required, although there are few studies addressing the role of dialysis in leptospirosis. There is currently no firm evidence regarding the place of immunological therapy, such as corticosteroids ${ }^{8}$ or immunoglobulins.

\section{Results and discussion}

\section{Methodology}

The main component of this research comprised a prospective study conducted in three hospitals in the Western Province of Sri Lanka, which is a high-prevalence area for leptospirosis. An analysis of hospital based sentinel surveillance data of leptospirosis over four years in Sri Lanka has confirmed that of nearly 4000 suspected cases $-47 \%$ were from this province. ${ }^{9}$ The study was conducted in the National Hospital of Sri Lanka (NHSL), Colombo North Teaching Hospital and Base Hospital Homagama. Data collection commenced in 2011, and is ongoing. All patients with potential leptospirosis (defined according to the World Health Organization [WHO] surveillance criteria 10) (Table 1) admitted to medical wards of these hospital were included in to the study.

\section{Table 1. Criteria for diagnosis of leptospirosis}

Acute febrile illness with headache, myalgia and prostration AND

$$
\begin{aligned}
& \text { EITHER } \\
& \text { Any of the following: } \\
& \text { - Conjunctival suffusion } \\
& \text { - Anuria or oliguria/haematuria } \\
& \text { - Cardiac arrhythmia or failure } \\
& \text { - Cough, haemoptysis and breathlessness } \\
& \text { - Haemorrhages } \\
& \text { - Meningeal irritation } \\
& \text { - Skin rash } \\
& \text { - Jaundice }
\end{aligned}
$$

OR history of exposure to leptospirosis

Microscopic Agglutination Test (MAT) was performed for laboratory confirmation of the disease. A positive result was considered as any one of the following: a) MAT titre of 400 or greater in single or paired samples, b) a four-fold increase in MAT titre between acute and convalescent serum samples, or c) seroconversion to a MAT titre greater than 200 between paired sampling. ${ }^{11}$ Confirmation of diagnosis was done retrospectively; those not meeting the diagnostic criteria were excluded from the leptospirosis cohort, and were categorized as 'non-leptospirosis fever'.

Data was collected from recruited patients with regards to their a) demographics, b) co-morbidities, c) past history of leptospirosis and d) clinical symptoms and signs during the current episode. The data was recorded in an interviewer-administered questionnaire. We did not intervene in the management of patients, but observed the clinical course of every patient by recording daily clinical status and results of haematological, biochemical and radiological investigations. The final outcome was recorded as death or recovery. Patients with any of the following were categorized as having severe leptospirosis; jaundice (bilirubin $>51.3 \mu \mathrm{mol} / \mathrm{L}$ ), renal insufficiency (oliguria with a urine output less than $400 \mathrm{ml}$ per day or creatinine $>133 \mu \mathrm{mol} / \mathrm{L}$ or blood urea $>25.5 \mathrm{mmol} /$ $\mathrm{L}$ ), and other indicators of poor outcome (intensive care unit stay, initiation of dialysis, hospital stay greater than 10 days and multi-organ dysfunction). ${ }^{12}$

Ethics clearance was obtained from the Ethics Review Committee of the Faculty of Medicine, University of Colombo (EC-12-056) and the Ethics Review Committee of the NHSL. Informed written consent was obtained from all participants prior to recruitment to the study.

\section{Challenges in diagnosis}

The clinical features of leptospirosis overlap with those of other tropical infectious diseases, such as dengue and rickettsial infections. We analysed the clinical features of leptospirosis in order to determine whether any of these predicted the development of severe leptospirosis. Data was obtained from 232 patients who met the clinical and laboratory criteria for leptospirosis, as defined earlier. This was one of the largest clinical cohorts of leptospirosis studied in Sri Lanka. The mean age of the sample was 42 years (range: 13-74 years, $\mathrm{SD} \pm 15)$. The male to female ratio was 7:1, in keeping with the known pattern of male predominance.

The clinical symptomatology of the sample is summarized in table 2. Fever was seen in all patients with leptospirosis. The other commonly reported symptoms were myalgia, headache, rigors and anorexia. Conjunctival suffusion was noted in approximately $53 \%$ of the sample. Neck stiffness and photophobia was reported from $21 \%$ and $12 \%$ respectively. Severe leptospirosis was seen in 56\%, and $35.5 \%$ needed haemodialysis for acute kidney injury (Table 3 ). The numbers requiring dialysis were high because these 
patients were predominantly from the National Hospital, Colombo, where patients are transferred in from other units for renal support, thus this proportion probably does not reflect the true incidence of acute kidney injury. Intensive care was required in $6.9 \%$, and the case fatality rate was $1.3 \%$. Penicillin and ceftriaxone were the most frequently used antibiotics. Three-fourths of all patients gave a history of contact with potentially contaminated water. Awareness of chemoprophylaxis was low, at 7\%, which was surprising, considering that the public health campaigns promote the use of doxycycline prophylaxis to farmers extensively.

Table 2. Clinical signs and symptoms

\begin{tabular}{lll}
\hline Symptom/sign & Number (\%) & $\begin{array}{l}\text { Mean day of } \\
\text { illness when sign } \\
\text { or symptom first } \\
\text { appeared }( \pm S D)\end{array}$ \\
\hline Fever & $221(100)$ & $1.1(0.61)$ \\
Myalgia & $188(85.1)$ & $1.1(0.43)$ \\
Headache & $182(82.4)$ & $1.2(0.72)$ \\
Rigors & $167(75.6)$ & $1.1(0.34)$ \\
Anorexia & $159(71.9)$ & $1.6(1.6)$ \\
Vomiting & $121(54.8)$ & $3.1(2.18)$ \\
Conjunctival suffusion & $117(52.9)$ & $2.9(1.98)$ \\
Arthralgia & $94(42.5)$ & $1.1(0.82)$ \\
Abdominal pain & $73(33.1)$ & $2.1(1.28)$ \\
Jaundice & $60(27.2)$ & $4.6(3.05)$ \\
Dyspnoea & $57(25.8)$ & $3.3(2.52)$ \\
Haematuria & $55(24.9)$ & $4.6(2.34)$ \\
Oliguria & $54(24.4)$ & $4.0(2.13)$ \\
Chest pain & $49(22.2)$ & $2.6(2.72)$ \\
Neck stiffness & $47(21.3)$ & $2.1(1.71)$ \\
Photophobia & $28(12.7)$ & $1.5(0.88)$ \\
Gastrointestinal bleeding & $23(10.4)$ & $6.1(2.98)$ \\
Haemoptysis & $4(1.8)$ & $6.0(3.92)$ \\
Skin rash & $2(0.9)$ & $2.0(1.41)$ \\
\hline
\end{tabular}

Table 3. Complications of leptospirosis

\begin{tabular}{ll}
\hline Complication & Number (\%) \\
\hline Acute kidney injury & $113(51.2)$ \\
Myocarditis & $5(2.3)$ \\
Shock & $5(2.3)$ \\
Acute liver failure & $3(1.4)$ \\
Acute respiratory distress syndrome & $2(0.9)$ \\
Pulmonary haemorrhage & $1(0.5)$ \\
Multi-organ dysfunction syndrome & $1(0.5)$ \\
\hline
\end{tabular}

We performed a multivariate binary regression analysis using correlation matrices to determine whether any clinical or investigation characteristics would be useful in differentiating leptospirosis from other similar febrile illnesses. ${ }^{13}$ The following were found to be independently associated with leptospirosis, when compared with other fevers with a similar clinical picture: history of exposure, serum creatinine $>150 \mu \mathrm{mol} / \mathrm{L}$, neutrophil differential percentage ${ }^{14}>82.8 \%$, platelet count ${ }^{14}$ $<85000 \mathrm{~mm}^{3}$, and serum bilirubin $>27 \mathrm{U} / \mathrm{L}$ (Nagelkerke R square - 0.567, Hosmer-Lemeshow test for goodness of fit - chi square $=2.898, p=0.941$ ). Notably, very few clinical or investigation characteristics reliably differentiate leptospirosis from non-leptospirosis fevers. This finding highlights the importance of developing more widely available rapid diagnostic tests for leptospirosis for use in hospitals with relatively limited laboratory facilities.

\section{Evaluation of two rapid diagnostic tests for leptospirosis}

Clinical leptospirosis is a biphasic illness, with a leptospiraemic phase occurring from the 4th to 7th day followed by a leptospiruric phase that can last for 4-30 days. Leptospiruria coincides with the immune phase which begins with the appearance of IgM antibodies. ${ }^{15}$ IgM based serology is of diagnostic value during this phase. Many rapid serodiagnostic assays are available, such as IgM based microplate enzyme-linked immunosorbant assay (ELISA), IgM dot ELISA test, IgM dipstick, latex agglutination test and haemagglutination assay. These are easy to perform and read, although their scientific validity with respect to sensitivity and specificity need further evaluation. To date, only one study in Sri Lanka has evaluated commercially available rapid immunodiagnostic kits. ${ }^{16}$ This study used the microplate ELISA (Institut Viron Serion $\mathrm{GmbH}$, Germany) and showed very low sensitivity and specificity values. Other rapid serological diagnostic assays have not been evaluated against a reference test in Sri Lanka. MAT is currently the standard immunological test used for diagnosis of leptospirosis in Sri Lanka. The MAT which is used in Sri Lanka detects both IgM and IgG, and is based on the strains Autumnalis, Grippotyphosa, Icterohaemorrhagica. Pomona, Pyogenes and Patoc. Laboratory confirmation based on MAT is a much delayed process as the test is only available in a central laboratory (the National Leptospirosis Reference Laboratory, MRI). Thus treatment is, in most instances, initiated on the basis of clinical assessment, with subsequent laboratory confirmation. In this study, the efficacy of two rapid immunodiagnostic assays for the detection of leptospira IgM antibodies, i.e., IgM based microplate ELISA (Diagnostic Automation, USA) and IgM based immunochromatography test (Leptocheck-WB test), together with MAT were compared. Since MAT detects crude antibodies (both IgM and IgG), the validity of using MAT as a gold standard was considered to be potentially imperfect, as MAT positivity could be a result of previous 
infection, while MAT negativity with tests of IgM alone being positive could be a result of MAT lacking sensitivity. In settings such as this, Bayesian latent class modelling (BLCM) has been suggested to be a better model, as it assumes that all tests are imperfect. We used the MICE (Modelling for Infectious diseases CEntre) ${ }^{17}$ tool for BLCM.

We evaluated these three diagnostic tests on 829 patients clinically suspected to have leptospirosis, based on the WHO diagnostic criteria detailed above. A MAT titer of $\geq 400$, anti-leptospiral $\lg M>20 \mathrm{IU} / \mathrm{ml}$, and presence of a red colour band in the test window were considered as positive for MAT, IgM ELISA and Leptocheck-WB test respectively. Patients with an alternative diagnosis $(n=21)$ and Hanta-virus infection (based on serology) $(n=8)$ were excluded. The analysis was performed using two methods, a) considering MAT as reference gold standard and b) using BLCM analysis. Positivity of MAT, IgM ELISA and Leptocheck-WB test were 33.4\%, 40.5\% and $47.4 \%$ respectively. The sensitivity and specificity of IgM ELISA ( $88.7 \%$ and $83.4 \%$ respectively) were higher than for Leptocheck-WB test (86.0\% and $71.8 \%)$ when MAT was used as reference standard. Bayesian latent class model analysis showed that MAT had the lowest sensitivity and highest specificity (76.6\% and $98.8 \%)$ whereas both parameters were high for IgM ELISA (90.4\% and $96.0 \%$ ) compared to Leptocheck-WB test (87.3\% and $81.9 \%$ ) (Table 4). ${ }^{18}$

Our findings show that MAT remains a suitable test for epidemiological screening, although its accuracy and reliability in acute leptospirosis infection requires further study, in particular with comparison against a gold standard such as PCR or culture. However, IgM ELISA is a suitable test for early diagnosis of acute leptospirosis infection. The Leptocheck-WB test is easier to perform, and could be recommended as a suitable test for rapid immuno-diagnosis in resource limited settings.

\section{A PIRO model for leptospirosis: a proposed prognostic score}

The PIRO model is a risk stratification system for severe sepsis. PIRO stands for predisposition $(P)$, infection (I), response (R) and organ dysfunction (O) ${ }^{19}$ similar to the TNM [tumor, nodes, metastases] classification for staging of malignancy.

In 2009, we performed a literature review with the aim of identifying prognostic indicators and indices of severity in leptospirosis, based on the PIRO model for sepsis. ${ }^{12}$ Although the aim of this review was to develop a scoring system for severity, available data was limited, and this was not possible. Nonetheless, several prognostic indicators were identified, and classified under the PIRO categories; we subsequently calculated odds ratios for relevant indicators (Figure 2). Clearly, the number of indicators were insufficient to be of practical relevance, however this review formed the basis of subsequent prospective studies aimed at identifying clinical and biochemical prognostic markers for leptospirosis.

Table 4. Prevalence, sensitivities and specificities, positive and negative predictive values (PPV and NPV) estimated by using gold standard model and Bayesian latent class model (BLCM)

\begin{tabular}{lll}
\hline Parameters & $\begin{array}{c}\text { MAT was assumed } \\
\text { as a perfect gold } \\
\text { standard }(\%)^{\star}\end{array}$ & $\begin{array}{l}\text { Bayesian latent } \\
\text { class model } \\
(\%)^{\star \star}\end{array}$ \\
\hline $\begin{array}{l}\text { Prevalence } \\
\text { MAT }\end{array}$ & $33.1(29.9-36.5)$ & $42.3(38.4-46.3)$ \\
Sensitivity & 100 & $76.6(70.9-81.9)$ \\
Specificity & 100 & $98.8(96.7-100)$ \\
PPV & 100 & $97.8(94.2-100)$ \\
NPV & 100 & $85.3(80.9-88.9)$ \\
Leptocheck & & $87.3(82.5-91.3)$ \\
Sensitivity & $86.0(81.1-89.9)$ & $81.9(77.8-85.4)$ \\
Specificity & $71.8(67.7-75.5)$ & $77.9(72.7-82.7)$ \\
PPV & $60.2(55.0-65.1)$ & $89.8(85.5-93.2)$ \\
NPV & $91.2(88.0-93.7)$ & $90.4(85.8-94.2)$ \\
IgM ELISA & $88.7(84.1-92.1)$ & $96.0(92.9-98.9)$ \\
Sensitivity & $83.4(79.9-86.4)$ & $94.3(89.8-98.5)$ \\
Specificity & $72.5(67.3-77.2)$ & $93.2(89.7-96.0)$ \\
PPV & $93.7(91.0-95.6)$ & \\
NPV &
\end{tabular}

* Gold standard model assumed that MAT is perfect $(100 \%$ sensitivity and $100 \%$ specificity; all patients with gold standard test positive are diseased and all patients with gold standard test negative are non-diseased). Values shown are estimated means with $95 \%$ confidence interval.

** Bayesian latent class model assumed that all tests evaluated are imperfect. Values shown are estimated median with $95 \%$ credible interval.

\begin{tabular}{|c|c|}
\hline Rredlellive faclor & Difference \\
\hline Older oge & MD B.00 5.40-10.61 \\
\hline Alcohol use & OR $4.431 .72-11.39$ \\
\hline Bacterosmia $>10^{4}$ & OR $133.002 .19-8081.94$ \\
\hline Rcised TNF alpho & OR $39.001 .86-817.63$ \\
\hline Tochyeardia & OR $9.994 .96-20.11$ \\
\hline Hypotension & OR $2.031 .40-2.95$ \\
\hline Trrombocytopenia & MD $31.26-39.64--22.88$ \\
\hline Leucocytos's & MD $2.321 .96-2.68$ \\
\hline Myocarditis & OR $119.005 .68-2494.85$ \\
\hline Afrythmics & OR $4.472 .94-6.79$ \\
\hline
\end{tabular}

Figure 2. Data from a literature review of prognostic markers in leptospirosis, categorized according to the PIRO model. 
We analysed our clinical data from 232 patients to determine whether any of these predicted the development of severe leptospirosis. Categorization of disease severity according to the criteria mentioned above showed that a majority of this sample $(130,56 \%)$ belonged to the severe category (table 3 ). This however may not be representative of the true incidence of severe leptospirosis as most of these patients were admitted to tertiary care referral centers, and included transfers from peripheral hospital for specialized care including renal support.

The case fatality rate was $1.3 \%$ ( 3 deaths). All three patients had acute kidney injury and one of them had acute liver injury as well. Since the mortality was low, we only compared clinical and demographic data with disease severity (mild vs. severe leptospirosis) to look for any statistically significant associations. Clinical features which had a direct bearing on classification of disease as mild and severe leptospirosis were excluded from this analysis (e.g. oliguria, jaundice) as that would have made the interpretations biased. Multivariate binary logistic regression analysis to control for confounding factors such as age, having diabetes mellitus, exposure risk factors (skin injury, contaminated water) was performed. Only the following laboratory features predicted severe leptospirosis: haematocrit $<29.8 \%(\mathrm{p}=0.011$, adjusted $\mathrm{OR}=3.750 ; \mathrm{Cl}=1.394-10.423)$, alanine ransaminase $>70 \mathrm{IU} / \mathrm{L}(\mathrm{p}=0.044$, adjusted $\mathrm{OR}=2.639 ; \mathrm{Cl}=1.028-6.774)$, and hyponatremia $<131 \mathrm{mEq} / \mathrm{L} \quad(p=0.019$, adjusted $\mathrm{OR}=6.413 ; \mathrm{Cl}=1.353-30.388$ ) (Nagelkerke $\mathrm{R}$ square $=0.697$, Hosmer Lemeshow goodness of fit-chi square $2.345, p=0.765)$. None of the clinical features predicted severe disease. Thus overall our data suggests that clinical or investigation data cannot be relied upon to predict disease severity in laboratory confirmed leptospirosis. ${ }^{20}$

\section{The need for biomarkers which could predict disease severity}

Since clinical features and standard biochemistry or haematology do not reliably predict which patients are likely to develop severe leptospirosis, there is a need to identify biochemical and genetic markers which are of prognostic value. Identification of such biomarkers is dependent on better understanding of the pathophysiology of leptospirosis. Currently, the pathophysiological basis of severe leptospirosis is poorly understood. In general, severe leptospirosis is thought to result from a systemic inflammatory vasculitis, ${ }^{21}$ which leads to multiple organ dysfunction. There has been much research into this subject over the past decade, and several potential biomarkers have already been earmarked for further investigation. ${ }^{22}$ These include biochemical molecules such as nitric oxide and its metabolites, ${ }^{23}$ cytokines such as IL-1, 6, TNF- $\alpha,{ }^{24}$ polymorphisms of human leukocyte antigen (HLA) and cytokine genes, ${ }^{25}$ human serum mannose binding lectin (which identifies pathogens activating the immune system), soluble ST2 receptors, long pentraxin PTX3 and copeptin. $^{26}$

\section{The role of oxidative stress in the pathogenesis of leptospirosis}

Oxidative stress has been postulated to play an important role in the pathogenesis of most severe inflammatory diseases. ${ }^{27,}{ }^{28}$ Reactive oxygen species (ROS)(superoxide anions, hydroxyl radical, singlet oxygen and hydrogen peroxide) are produced in large quantities during the respiratory burst. ${ }^{29}$ Similarly, reactive nitrogen species (RNS) (nitric oxide, nitrites and nitrates) are produced as a result of enhanced expression of inducible nitric oxide synthase. While the natural role of these substances is to provide protection against pathogens, an exaggerated response is thought to result in tissue and organ damage, and could contribute to the development of severe manifestations of leptospirosis. Both ROS and RNS cause damage to protein and lipid components of cells (Figure 3).

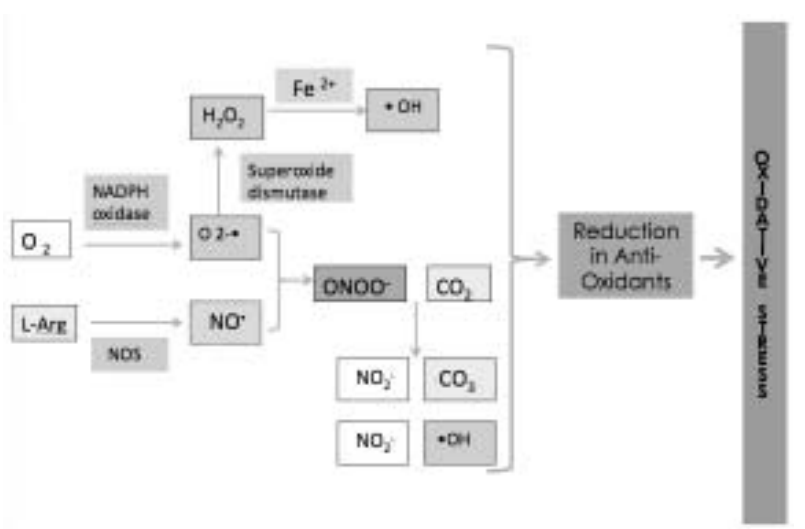

Figure 3. Oxidative stress caused by reactive oxygen species (ROS) and reactive nitrogen species.

During health, there is a fine balance between prooxidants and anti-oxidants. We postulated that this balance between pro-oxidants and anti-oxidants is deranged in severe leptospirosis, resulting in organ damage and multi-organ failure (Figure 4).

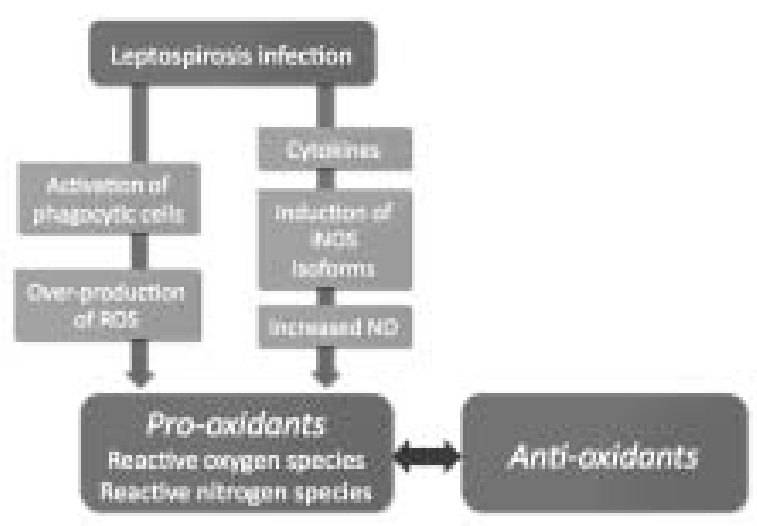

Figure 4. Suggested model for mechanisms of tissue damage resulting from oxidative stress in leptospirosis. ROSreactive oxygen substances, NO - nitric oxide. 
Oxidant induced damage to amino acids, in particular lysine, arginine and proline, gives rise to reactive aldehydes and ketones, known as protein carbonyls $^{30,31}$. Protein carbonyl levels in serum can be measured, and are a reliable indicator of the degree of protein damage induced by oxidative stress. ${ }^{32}$ Oxidative damage to lipids causes degradation or decomposition of lipids, resulting in the production of lipid hydroperoxide. ${ }^{33}$ Measurement of lipid peroxide provides an overall index of oxidative stress induced damage to lipids.

Oxidative stress is counteracted by anti-oxidants in blood. Glutathione, albumin, uric acid and bilirubin are common anti-oxidants, and play a vital role in maintaining the balance between pro-oxidants and anti-oxidants. Raised levels of pro-oxidants (RNS and ROS) causes anti-oxidants in blood to be consumed, resulting in diminished anti-oxidant capacity. ${ }^{34}$ While direct determination of ROS and RNS in vivo is difficult, overall anti-oxidant capacity can be measured in serum, and provides an indirect indication of oxidative stress and the potential for protection against oxidative damage..$^{35}$

We thus sought to determine whether markers of oxidative stress was higher in patients with severe leptospirosis, and whether there was evidence of tissue damage in such patients. We sought to demonstrate the effects of oxidative stress by two methods:

1. Measurement of markers of oxidative damage to proteins and lipids, by measurement of protein carbonyl and lipid peroxide levels

\section{Measurement of serum anti-oxidant capacity.}

We measured lipid peroxide levels and protein carbonyl levels in 110 patients with leptospirosis. Of them, 60 patients had severe leptospirosis, based on the definitions given above. We also included 30 patients with critical phase dengue, and 30 healthy controls (table 5). ${ }^{36}$

Lipid peroxide levels were measured using the Ferrous Oxidation-Xylenol orange (FOX2) assay. Our findings showed that lipid peroxide levels were high in patients with leptospirosis, and were low in patients with dengue and healthy controls. While this indicated that oxidative stress induced damage to lipids takes place in leptospirosis, there was no significant difference in lipid peroxide levels in severe and mild disease. Lipid peroxide levels in dengue were low, and similar to healthy controls (Figure 5).

Protein carbonyl levels were measured in the same patients using a commercial protein carbonyl kit. Protein carbonyl levels were significantly higher in severe leptospirosis compared to mild leptospirosis (Figure 6). These results indicated that, while greater oxidative damage to proteins takes place in leptospirosis, this damage is significantly greater in patients with severe leptospirosis. In dengue, protein carbonyl levels were similar to healthy controls.

We measured serum anti-oxidant capacity using the ABTS (2,2'-azinobis-3-ethylbenzothiazoline-6-sulfonic acid) method. Antioxidant capacity was significantly diminished in both severe and mild leptospirosis, and also in dengue, compared with healthy controls (Figure 7).

Overall, these finding show that oxidative stress is high in leptospirosis, and results in damage to proteins and lipids. Interestingly, damage to lipids, while higher in leptospirosis compared with dengue and healthy controls, is not significantly higher in severe leptospirosis when compared with mild leptospirosis. On the other hand, damage to proteins is significantly greater in severe leptospirosis. This could be due to the greater amounts of protein in serum, while lipids are present mostly in tissues. Nonetheless, damage to proteins is of importance; oxidative damage to proteins can result in significant injury to tissues, and can disrupt enzymatic processes within the cells. Measurement of protein carbonyl levels may have a place in differentiating severe from mild forms of leptospirosis. The finding that antioxidant capacity is similar in mild and severe forms of leptospirosis cannot be fully explained as yet. One possibility is that the high bilirubin and uric acid levels, which parallel the hepatic and renal dysfunction which occurs in severe leptospirosis, act as anti-oxidant buffers, thus resulting in equalisation of anti-oxidant capacity in mild and severe disease.

Table 5. Characteristics of patients in whom markers of oxidative stress were measured

\begin{tabular}{lcccc}
\hline & $\begin{array}{c}\text { Severe leptospirosis } \\
(S L)\end{array}$ & $\begin{array}{c}\text { Mild leptospirosis } \\
(M L)\end{array}$ & $\begin{array}{c}\text { Dengue } \\
(\text { DC) }\end{array}$ & $\begin{array}{c}\text { Healthy Controls } \\
(H C)\end{array}$ \\
\hline Number of patients & 60 & 50 & 30 & 30 \\
Mean age \pm SD (years) & $40.6 \pm 14.00$ & $37.7 \pm 14.7$ & $39.41 \pm 18.29$ & $38.1 \pm 12.23$ \\
Gender (male:female) & 53.7 & $43: 7$ & 26.4 & 26.4 \\
\hline
\end{tabular}




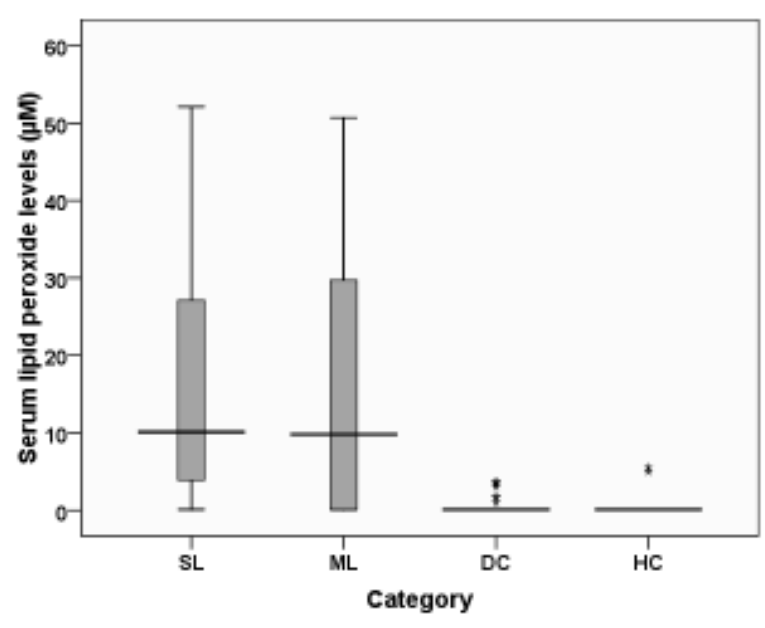

Figure 5. Lipid peroxide levels in patients with leptospirosis, dengue, and healthy controls.

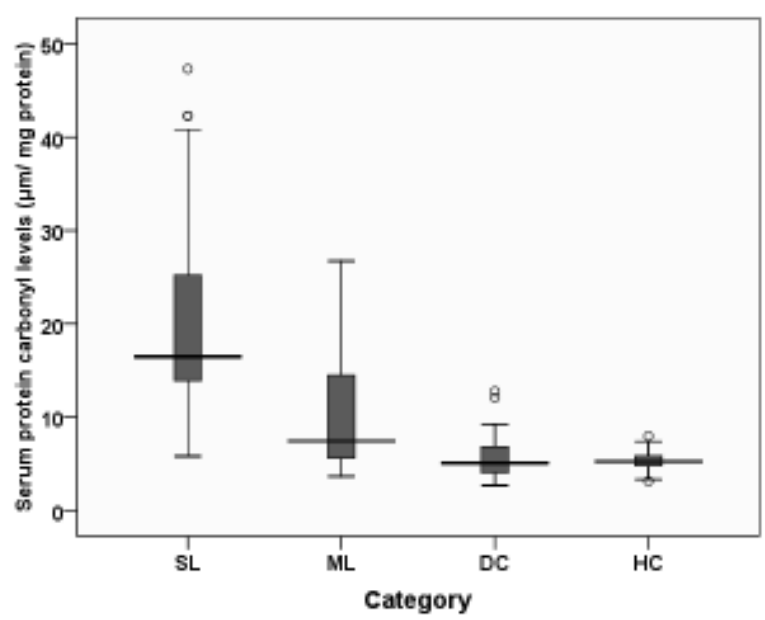

Figure 6. Protein carbonyl levels in patients with leptospirosis, dengue, and healthy controls.

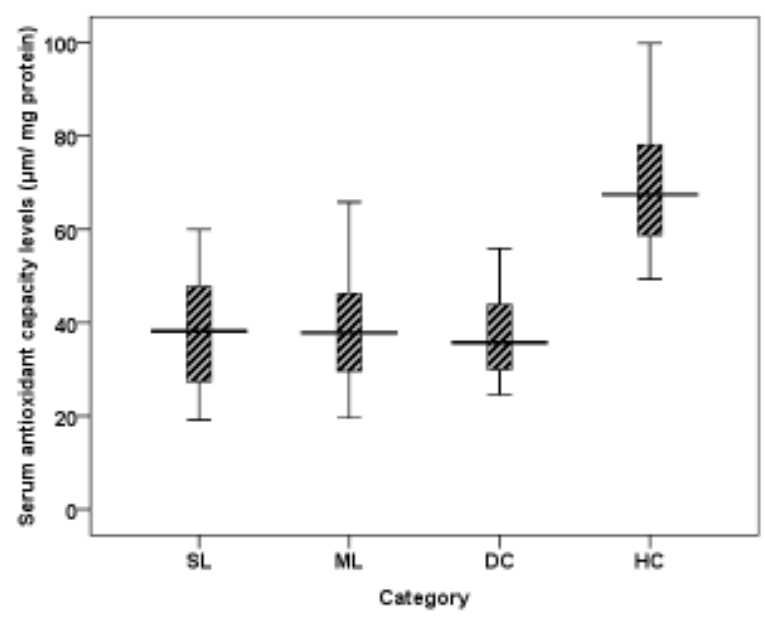

Figure 7. Serum anti-oxidant capacity in patients with leptospirosis, dengue and healthy controls.

\section{Nitric oxide: mediator or marker of oxidative stress?}

Nitric oxide (NO) is an important biological free radical which is involved in basic physiological functions; it has both cytoprotective and cytotoxic properties. ${ }^{37,38}$ This extremely volatile gas is synthesized from L-arginine by the isoforms of nitric oxide synthase (NOS) enzyme, in the vascular endothelium. It is quickly oxidized into nitrites $\left(\mathrm{NO}^{2-}\right)$ and then nitrates $\left(\mathrm{NO}^{3-}\right) .^{39}$ In the presence of oxygenated haemoglobin, conversion from $\mathrm{NO}^{2-}$ to $\mathrm{NO}^{3-}$ occurs in less than an hour. ${ }^{40} \mathrm{NO}^{2-}$ has been frequently used as a marker for NO production. ${ }^{41}$

Leptospirosis is known to stimulate the production of pro-inflammatory cytokines ${ }^{42}$ which could result in elevated NO levels ${ }^{43}$. Previous studies on leptospirosis and malaria have opened up a debate as to how NO contributes to the pathogenesis of severe illness in these conditions. ${ }^{44,45} \mathrm{It}$ is unclear whether overproduction of $\mathrm{NO}$ is a primary pathogenic mechanism in the causation of severe leptospirosis, or whether NO plays a protective role in severe disease. Nonetheless, high levels of NO contributes to oxidative stress, through the creation of reactive nitrogen species (RNS). ${ }^{27,28}$ (Figure 3).

In a study of 40 patients with leptospirosis, 26 of whom had severe disease, we demonstrated that total nitrite and nitrate levels were significantly higher in patients with severe leptospirosis, compared to those with mild disease, healthy controls, and non-leptospirosis fever. ${ }^{23}$ There has been much debate as to whether uncorrected levels of nitrate/nitrite (NOx) are a reliable index of NO activity. In malaria, high NOx levels have been shown to be associated with worse prognosis. ${ }^{46,47}$ However, many patients with severe illness have deranged renal function. NOx is excreted by the renal route and it has been suggested that NOx levels should be corrected for renal function. In malaria, when NOx levels were corrected for renal function using the formula NOx/ creatinine, it was shown that corrected NOx levels were actually lower in patients with severe malaria. ${ }^{48,49}$ Since renal impairment occurs in over half the patients with leptospirosis, and was nearly always present in severe leptospirosis, we applied the same principle to our cohort of patients. Strikingly, when NOx was corrected for renal function, NOx levels were significantly lower in patients with severe leptospirosis, and were similar to levels seen in healthy controls, while corrected NOx levels were high in mild leptospirosis (Figure 8).

In a larger prospective study, we compared nitrite and nitrate levels among four groups; severe leptospirosis (SL), mild leptospirosis (ML), non-leptospirosis fever (NLF) and healthy controls (HC), with 60 subjects in each group. The Griess assay was used to measure NOx levels. Patients with severe leptospirosis were found to have significantly higher nitrite levels when compared with those with mild disease, patients with other fevers, and healthy controls (Figure 9). 


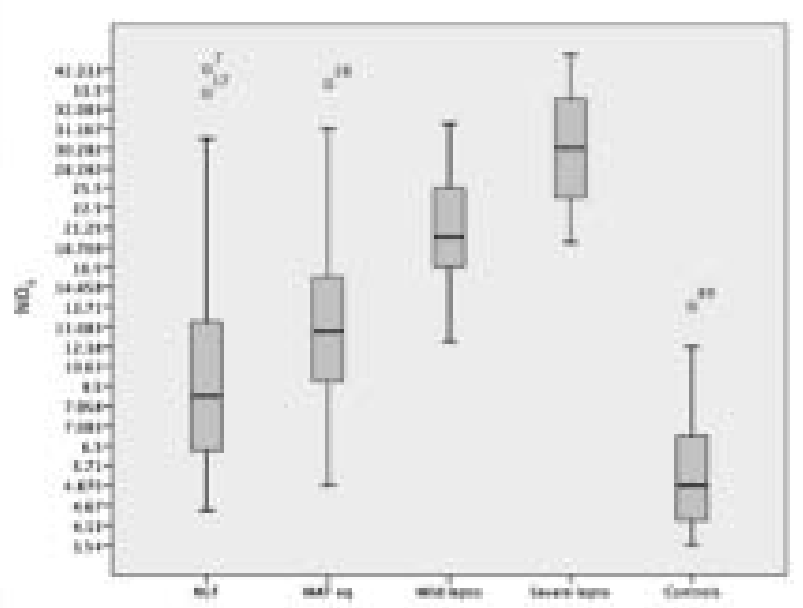

Uncorrected NOx

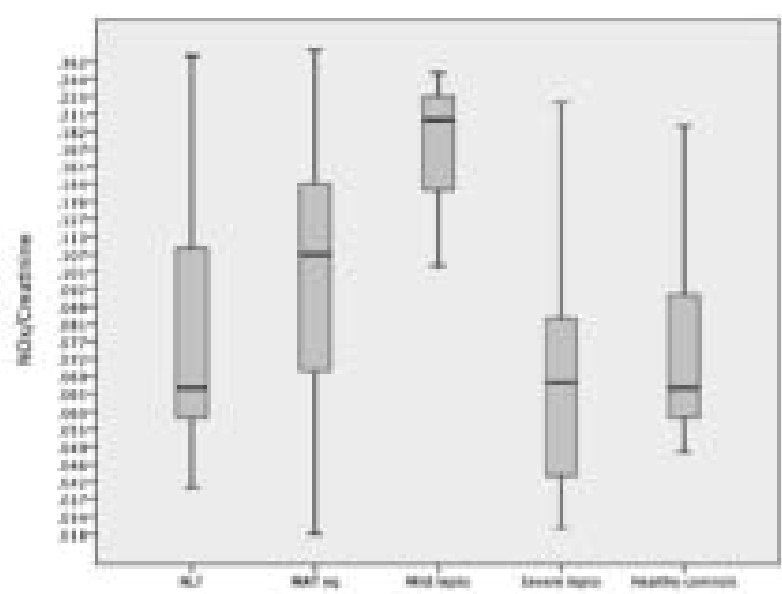

Corrected NOX

Figure 8. When corrected for renal function, NOx levels are lowest in patients with severe leptospirosis. From Kalugalage et al (2013). ${ }^{23}$

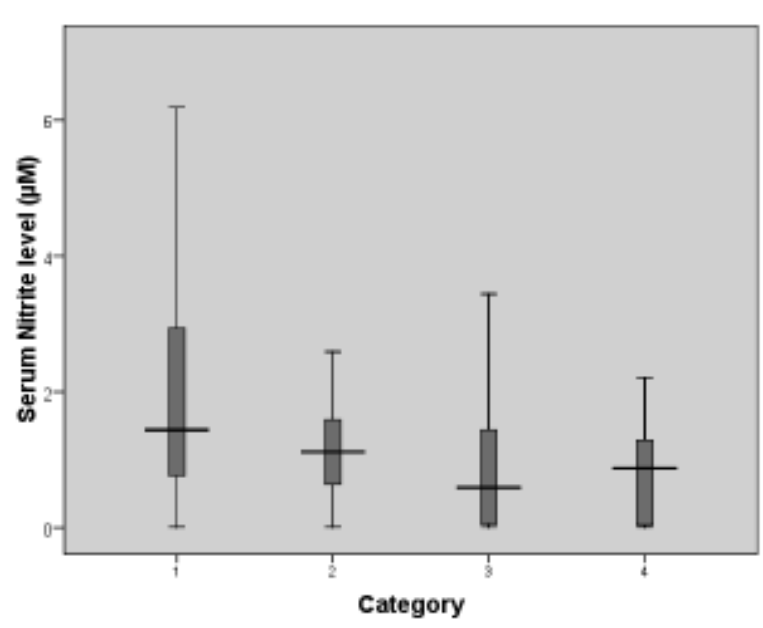

Figure 9. Serum nitrite levels in severe (1) and mild leptospirosis (2), non-leptospirosis fever (3) and healthy controls (4).

Nitrite levels appear to be independent of renal function, possibly because its transient nature, and thus elevated nitrite levels are potentially a marker of severity in leptospirosis. Nitrite levels over $2 \mu \mathrm{M}$ appear to predict the development of severe disease in patients with leptospirosis. Based on these findings, we developed a rapid colorimetric test of serum nitrite levels, which could be used in hospitals with limited resources. This simple test used a colorimetric comparison of different dilution of serum with a test concentration of nitrite of $2 \mu \mathrm{M}$. We suggest that this simple test could be of practical value to clinicians in the periphery, to predict which patients were likely to develop severe disease.

\section{Conclusions}

In this series of studies, we demonstrated that IgM based rapid diagnostic tests are reasonably reliable and accurate. We also showed that clinical parameters are unreliable as predictors of severity in leptospirosis. Oxidative stress appears to be high in patients with leptospirosis, and damage to proteins and lipids appears to take place; it is likely that this oxidant induced damage plays a role in tissue and organ dysfunction. Protein damage seems to be greater in patients with severe leptospirosis. Nitric oxide plays a complex role in the pathogenesis of severe disease, and appear to be elevated in patients with severe leptospirosis. Serum nitrite levels show promise as a serum marker which could predict the development of severe disease, and appear independent of renal function. A rapid assay of serum nitrite may have potential use in hospitals with limited resources.

\section{Future directions}

In 2013, we established a Tropical Medicine Research Unit (TMRU) under the Faculty of Medicine, University of Colombo, in order to strengthen and continue this work. The unit is located in the Clinical Medicine Academic and Research Centre, National Hospital Complex, Colombo. The unit collaborates with the University of Oxford, UK, and the Mahidol-Oxford Research Unit, Thailand. TMRU will continue its collaborations with the IBMBB, the MRI, and with several clinicians in hospitals in the country. Many new collaborative research projects are planned. Future focus will be directed towards investigating other derangements in humoral and cellular immunity in leptospirosis, and towards determining sero-prevalence of leptospirosis in different districts in Sri Lanka, with mapping of cases using Geographic Information Systems (GIS) technology. Based on the hypothesis that oxidative stress may play a role in the causation of severe leptospirosis, a randomised controlled trial of $\mathrm{N}$-acetyl cysteine, an antioxidant, in the treatment of severe leptospirosis is about to commence. 


\section{Acknowledgements}

These studies were the result of the collaborative efforts of a large team of researchers from several institutions: Professor Rajitha Wickramasinghe, Professor of Community Medicine, University of Kelaniya; Dr Gayani Premawansa, Consultant Physician, North Colombo Teaching Hospital; Dr Rohini Gunaratna, Ms Shalini Wickramasinghe, Ms Egwin Eugene, MSc Immunology students; Dr Hasith Wickramasinghe and Dr Nandana Dikmadugoda, Consultant Physicians, Base Hospital Homagama; Dr Chaturaka Rodrigo, Lecturer, Department of Clinical Medicine, University of Colombo, Dr Tharanga Fernando, Dr Nipun Lakshitha De Silva, Dr Sachith Maduranga, Dr Nuwanthi Nandasiri, and Dr S. Sarangan, Demonstrators, Tropical Medicine Research Unit, Faculty of Medicine, University of Colombo. The Consultants, doctors and other staff of the National Hospital, Colombo North Teaching Hospital, Base Hospital Homagama, IBMBB, and MRI are gratefully acknowledged for their help and continued support. The patients who participated in the study are gratefully acknowledged. The National Research Council and National Science Foundation are acknowledged with gratitude for providing funding for this study. Professor Rohan Jayasekara, Dean Faculty of Medicine is gratefully acknowledged for providing constant support towards the establishment of the Tropical Medicine Research Unit.

\section{Funding}

Funding for these studies was primarily obtained from the National Science Foundation, Sri Lanka, Grant No. NSF/2011/HS/19, and the National Research Council, Sri Lanka, Grant No: NRC 12-077. In addition, funding and support was received from the MSc Immunology Programme of the IBMBB, University of Colombo.

\section{References}

1. Pappas G, Papadimitriou P, Siozopoulou V, Christou L and Akritidis N. The globalization of leptospirosis: worldwide incidence trends. Int J Infect Dis. 2008; 12: 351-7.

2. Vijayachari P, Sugunan AP, Umapathi T, Sehgal SC. Evaluation of darkground microscopy as a rapid diagnostic procedure in leptospirosis. The Indian Journal of Medical Research 2001; 114: 54-8.

3. Epidemiology Unit. Leptospirosis cases by month. Colombo: Ministry of Health Sri Lanka, 2013.

4. Rajapakse S, Rodrigo C, Balaji K and Fernando SD. Atypical manifestations of leptospirosis. Transactions of the Royal Society of Tropical Medicine and Hygiene 2015; 109: 294-302.

5. Spichler AS, Vilaca PJ, Athanazio DA, et al. Predictors of lethality in severe leptospirosis in urban Brazil. The American Journal of Tropical Medicine and Hygiene 2008; 79: 911-4.
6. Rajapakse S. Diagnosing and predicting outcome in leptospirosis: the need for clinically relevant basic sciences research. The Ceylon Medical Journal 2014; 59: 115-7.

7. Brett-Major DM and Coldren R. Antibiotics for leptospirosis. The Cochrane Database of Systematic Reviews. 2012; 2: CD008264.

8. Rodrigo C, Lakshitha de Silva N, Goonaratne R, et al. High dose corticosteroids in severe leptospirosis: a systematic review. Transactions of the Royal Society of Tropical Medicine and Hygiene 2014; 108: 743-50.

9. Gamage CD, Amarasekera J, Palihawadana P, et al. Analysis of hospital-based sentinel surveillance data on leptospirosis in Sri Lanka, 2005-2008. Japanese Journal of Infectious Diseases 2012; 65: 157-61.

10. World Health Organization. Human Leptospirosis. Guidance for diagnosis surveillance and control. Geneva: World Health Organization, 2003.

11. World Health Organization. Report of the Second Meeting of the Leptospirosis Burden Epidemiology Reference Group. Geneva: World Health Organization, 2011.

12. Rajapakse S, Rodrigo $C$ and Haniffa R. Developing a clinically relevant classification to predict mortality in severe leptospirosis. Journal of Emergencies, Trauma, and Shock 2010; 3: 213-9.

13. Rajapakse $S$, Weeratunga $P$, Niloofa $R$, et al. A Diagnostic Scoring Model for Leptospirosis in Resource Limited Settings. PLoS Neglected Tropical Diseases 2016; 10: e0004513.

14. De Silva NL, Niloofa M, Fernando N, et al. Changes in full blood count parameters in leptospirosis: a prospective study. International Archives of Medicine 2014; 7: 31.

15. Gunawardhana SA and Sellahewa KH. Clinical features of leptospirosis: a prospective descriptive study at the National Hospital of Sri Lanka (NHSL) in 2007. The Ceylon Medical Journal 2008; 53: 155-6.

16. Reller ME, Bodinayake C, Nagahawatte A, et al. Leptospirosis as frequent cause of acute febrile illness in southern Sri Lanka. Emerging Infectious Diseases 2011; 17: 1678-84.

17. Modelling for Infectious Diseases Centre. http:// mice.tropmedres.ac/home.aspx. Accessed 1st December 2014.

18. Niloofa R, Fernando N, de Silva NL, et al. Diagnosis of Leptospirosis: Comparison between Microscopic Agglutination Test, IgM-ELISA and IgM Rapid Immunochromatography Test. PloS One 2015; 10: e0129236.

19. Rubulotta F, Marshall JC, Ramsay G, Nelson D, Levy M and Williams M. Predisposition, insult/infection, response, and organ dysfunction: A new model for staging severe sepsis. Critical Care Medicine 2009; 37: 1329-35.

20. Rajapakse S, Weeratunga P, Niloofa MJ, et al. Clinical and laboratory associations of severity in a Sri Lankan cohort of patients with serologically confirmed leptospirosis: a prospective study. Transactions of the Royal Society of Tropical Medicine and Hygiene 2015; 109: $710-6$. 
21. Medeiros Fda R, Spichler A and Athanazio DA. Leptospirosis-associated disturbances of blood vessels, lungs and hemostasis. Acta tropica 2010; 115: 155-62.

22. Rajapakse S, Rodrigo C, Handunnetti SM and Fernando SD. Current immunological and molecular tools for leptospirosis: diagnostics, vaccine design, and biomarkers for predicting severity. Annals of Clinical Microbiology and Antimicrobials 2015; 14: 2.

23. Kalugalage $T$, Rodrigo $C$, Vithanage $T$, et al. Low serum total nitrite and nitrate levels in severe leptospirosis. BMC Infectious Diseases 2013; 13: 206.

24. Niwattayakul K, Homvijitkul J, Niwattayakul S, Khow O and Sitprija V. Hypotension, renal failure, and pulmonary complications in leptospirosis. Ren Fail 2002; 24: 297-305.

25. Fialho RN, Martins L, Pinheiro JP, et al. Role of human leukocyte antigen, killer-cell immunoglobulin-like receptors, and cytokine gene polymorphisms in leptospirosis. Hum Immunol 2009; 70: 915-20.

26. Miranda KA, Vasconcelos LR, Coelho LC, Lima Filho JL, Cavalcanti MS and Moura P. High levels of serum mannose-binding lectin are associated with the severity of clinical signs of leptospirosis. Braz J Med Biol Res 2009; 42: 353-7.

27. Winterbourn CC. Reconciling the chemistry and biology of reactive oxygen species. Nature Chemical Biology 2008; 4: 278-86.

28. Kyaw M, Yoshizumi M, Tsuchiya K, Izawa Y, Kanematsu Y and Tamaki T. Atheroprotective effects of antioxidants through inhibition of mitogen-activated protein kinases. Acta Pharmacologica Sinica 2004; 25: 977-85.

29. Girotti AW. Lipid hydroperoxide generation, turnover, and effector action in biological systems. Journal of Lipid Research 1998; 39: 1529-42.

30. Berlett BS and Stadtman ER. Protein oxidation in aging, disease, and oxidative stress. The Journal of Biological Chemistry 1997; 272: 20313-6.

31. Dean RT, Fu S, Stocker R and Davies MJ. Biochemistry and pathology of radical-mediated protein oxidation. The Biochemical Journal 1997; 324 (Pt 1): 1-18.

32. Dalle-Donne I, Rossi R, Giustarini D, Milzani A and Colombo R. Protein carbonyl groups as biomarkers of oxidative stress. Clinica Chimica Acta; International Journal of Clinical Chemistry 2003; 329: 23-38.

33. Halliwell B, Chirico S. Lipid peroxidation: its mecha-nism, measurement, and significance. The American Journal of Clinical Nutrition 1993; 57: 715S-24S; discussion 24S$25 S$.

34. Goodyear-Bruch C, Pierce JD. Oxidative stress in critically ill patients. American journal of critical care: an official publication, American Association of Critical-Care Nurses 2002; 11: 543-51; quiz 52-3.

35. Arguelles S, Garcia S, Maldonado M, Machado A and Ayala A. Do the serum oxidative stress biomarkers provide a reasonable index of the general oxidative stress status? Biochimica et Biophysica Acta 2004; 1674: 251-9.
36. Fernando N, Wickremesinghe S, Niloofa R, et al. Protein Carbonyl as a Biomarker of Oxidative Stress in Severe Leptospirosis, and Its Usefulness in Differentiating Leptospirosis from Dengue Infections. PloS One. 2016; 11: e0156085.

37. Cooke JP, Tsao PS. Cytoprotective effects of nitric oxide. Circulation 1993; 88: 2451-4.

38. Moncada S, Higgs A. The L-arginine-nitric oxide pathway. The New England Journal of Medicine 1993; 329: 2002-12.

39. Ghasemi A, Zahedi AsI S, Mehrabi Y, Saadat N and Azizi F. Serum nitric oxide metabolite levels in a general healthy population: relation to sex and age. Life Sciences 2008; 83: 326-31.

40. Moncada S, Palmer RM, Higgs EA. Nitric oxide: physiology, pathophysiology, and pharmacology. Pharma-cological Reviews 1991; 43: 109-42.

41. Kelm M, Preik-Steinhoff H, Preik M, Strauer BE. Serum nitrite sensitively reflects endothelial NO formation in human forearm vasculature: evidence for biochemical assessment of the endothelial L-arginine-NO pathway. Cardiovascular Research 1999; 41: 765-72.

42. Cinco $M$, Vecile E, Murgia R, Dobrina $P$ and Dobrina $A$. Leptospira interrogans and Leptospira peptidoglycans induce the release of tumor necrosis factor alpha from human monocytes. FEMS Microbiology Letters 1996; 138: 211-4.

43. Yang GG, Hsu YH. Nitric oxide production and immunoglobulin deposition in leptospiral hemorrhagic respiratory failure. Journal of the Formosan Medical Association = Taiwan Yi Zhi 2005; 104: 759-63.

44. Maciel EA, Athanazio DA, Reis EA, et al. High serum nitric oxide levels in patients with severe leptospirosis. Acta Tropica 2006; 100: 256-60.

45. Gramaglia I, Sobolewski P, Meays D, et al. Low nitric oxide bioavailability contributes to the genesis of experimental cerebral malaria. Nature Medicine 2006; 12: 1417-22.

46. Al Yaman FM, Mokela D, Genton B, Rockett KA, Alpers MP and Clark IA. Association between serum levels of reactive nitrogen intermediates and coma in children with cerebral malaria in Papua New Guinea. Transactions of the Royal Society of Tropical Medicine and Hygiene 1996; 90: $270-3$

47. Kremsner PG, Winkler S, Wildling E, et al. High plasma levels of nitrogen oxides are associated with severe disease and correlate with rapid parasitological and clinical cure in Plasmodium falciparum malaria. Transactions of the Royal Society of Tropical Medicine and Hygiene 1996; 90: 44-7.

48. Anstey NM, Weinberg JB, Hassanali MY, et al. Nitric oxide in Tanzanian children with malaria: inverse relationship between malaria severity and nitric oxide production/nitric oxide synthase type 2 expression. The Journal of Experimental Medicine 1996; 184: 557-67.

49. Anstey NM, Granger DL, Weinberg JB. Nitrate levels in malaria. Transactions of the Royal Society of Tropical Medicine and Hygiene 1997; 91: 238-40. 